\title{
Humoral and cellular immune responses to Blomia tropicalis and concanavalin A-binding fractions in atopic patients
}

\author{
R. Alves ${ }^{1}$, D.A.O. Silva1 ${ }^{1}$, J.F.C. Fernandes ${ }^{1}$, K.C. Almeida ${ }^{1}$, L.H. Ynoue 1 , \\ C.T.V. Bernardes ${ }^{1}$, P.F.S. Moreira ${ }^{1}$, M.L. Gennari-Cardoso' ${ }^{2}$, S.J. Sung ${ }^{3}$ and \\ E.A. Taketomi ${ }^{1}$
}

${ }^{1}$ Laboratório de Alergia e Imunologia Clínica, Instituto de Ciências Biomédicas, Universidade Federal de Uberlândia, Uberlândia, MG, Brasil

2Divisão de Imunologia e Microbiologia, Departamento de Ciências Biológicas, Universidade Estadual de Santa Cruz, Ilhéus, BA, Brasil

${ }^{3}$ Department of Medicine, Center for Immunity, Inflammation and Regenerative Medicine, University of Virginia, Charlottesville, VA, USA

Correspondence to: E.A. Taketomi, Laboratório de Alergia e Imunologia Clínica, ICBIM, UFU, Av. Pará, 1720, Bloco 4C, Campus Umuarama, 38400-902 Uberlândia, MG, Brasil

Fax: +55-34-3232-8620. E-mail: eat4y@yahoo.com.br

Blomia tropicalis, Dermatophagoides pteronyssinus and $D$. farinae are prevalent house dust mites. Concanavalin A-binding components derived from $B$. tropicalis (Bt-ConA extract) are highly immunogenic in allergic diseases. The aim of the present study was to evaluate the humoral and cellular immune responses to $B$. tropicalis in mite-sensitized patients. A total of 137 patients with allergic rhinitis with/without asthma and 109 non-atopic subjects were selected and analyzed by the skin prick test, and for total serum IgE and specific IgE levels to both Bt-total and Bt-ConA extracts, their proliferative response and cytokine (IFN- $\gamma$ and IL-5) production by peripheral blood mononuclear cells (PBMC) stimulated with both extracts. Skin prick test showed that $70 \%$ of the patients were sensitized to $\mathrm{Bt}(\mathrm{Bt}+)$ and similar levels of specific lgE to Bt-total and $\mathrm{Bt}$-ConA extracts were demonstrable in $\mathrm{Bt}+$ patients. Significant PBMC proliferation was observed in response to Bt-total extract in $\mathrm{Bt}+$, but not in $\mathrm{Bt}-$ patients and non-atopic subjects $(P<0.001)$. Bt-ConA extract induced increased proliferative responses in all patient groups compared to medium alone $(P<0.05)$, but these responses were significantly decreased in the presence of the mannopyranoside ConA inhibitor $(P<0.05)$. Significant IFN- $\gamma$ production was observed after Bt-ConA stimulation of $B t+p a t i e n t s ~(P<0.05)$, while Bt-total extract had no effect. IL-5 production was consistently detected in $\mathrm{Bt}+$ patients after allergen-specific stimulation or with no stimulus, indicating that PBMC from allergic patients are prone to produce Th2 profile cytokines, spontaneously or inductively by allergen restimulation. These data showed that ConA-binding components isolated from $B$. tropicalis may contain relevant antigens that are involved in both humoral and cellular immune responses. However, without an additional purification procedure to eliminate the residual contamination with ConA, its use in immunotherapeutic procedures cannot be recommended.

Key words: Blomia tropicalis; Concanavalin A; Cellular proliferation; IFN- $\gamma$; IL-5; Immunoglobulin E

Research supported by CAPES (\#503-313/COIPA), CNPq (\#479577/2004-8), and FAPEMIG (\#CDS 1605/05).

Received January 31, 2008. Accepted August 26, 2008

\section{Introduction}

In tropical and subtropical climates, Blomia tropicalis (Bt), Dermatophagoides pteronyssinus (Dp) and D. farinae (Df) are prevalent house dust mites (HDMs) $(1,2)$. HDMs have been recognized as important sources of allergens for the development of allergic diseases, particularly asthma and allergic rhinitis (3). Sensitization to indoor allergens is the most important independent risk factor associated with asthma, and positive skin prick tests (SPT) or serum IgE 
antibodies to HDMs are demonstrable in allergic patients (4).

Glycosylated antigens play a crucial role in different pathologies and are highly immunogenic (5-7). Mannanrich glycosylated components derived from Candida albicans have been recognized by $\operatorname{lgE}$ antibodies in patients with allergic manifestations including asthma, rhinitis, and atopic dermatitis (8). Recently, we reported IgE, IgG1 and IgG4 responses to $B$. tropicalis in patients with respiratory allergy using Bt-total extract and concanavalin A-binding components derived from $B$. tropicalis (Bt-ConA extract) (9). The results of that study showed that these patients seem to be more frequently sensitized to $B$. tropicalis highmolecular weight (54-104 kDa) components, mostly present in Bt-ConA extract than to the low-molecular weight (11-15 kDa) allergens, which have been demonstrated primarily in other reports $(10,11)$.

Several investigators have shown consistently in both human and mouse models that Th2 cytokines (IL-4, IL-5 and IL-13) are the main contributors to the development of allergic diseases by stimulating $B$ cells to produce $\lg E$ antibodies, recruitment and activation of eosinophils, and mucus production. Interferon- $\gamma$ (IFN- $\gamma$ ), a Th1 cytokine profile, acts in conjunction with Th2 cytokines to maintain chronic allergic inflammation (12-14).

The aims of the present study were to evaluate the humoral and cellular immune responses to $B$. tropicalis in mite-sensitized patients by determining the levels of total serum $\lg \mathrm{E}$ and specific IgE to Bt-total and Bt-ConA extracts, the proliferative response and the cytokine (IFN- $\gamma$ and IL-5) production by peripheral blood mononuclear cells (PBMC) stimulated with both allergen extracts.

\section{Subjects and Methods}

A total of 137 patients (54 males and 83 females; mean age \pm SD: $27.6 \pm 9.4$ years) with perennial allergic rhinitis with/without intermittent or persistent, mild-to-moderate asthma were selected for the study at the Allergy and Clinical Immunology Unit, Federal University of Uberlândia, Uberlândia, MG, Brazil, based on their history of respiratory symptoms related to house dust exposure and physical examination (15-17). The presence of upper airway infections in the last 30 days prior to the study, the use of antihistaminic drugs earlier in the week, and the use of oral or topic corticosteroids in the previous 4 weeks were used as exclusion criteria. As the control group, 109 healthy subjects (37 males and 72 females; mean age \pm SD: 31.1 \pm 11.7 years), with no history of allergic diseases and a negative SPT to all aeroallergen extracts tested were included. The study was approved by the Ethics Committee in Human Research of the Federal University of
Uberlândia and written informed consent was obtained from all participants.

\section{Skin prick test and serum samples}

All individuals underwent SPT with the following allergen extracts: mite (B. tropicalis, $D$. pteronyssinus and $D$. farinae) extracts prepared as described in Ref. 18, and commercial extracts of cockroach (Blattella germanica and Periplaneta americana), molds (Cladosporium spp) and pet danders (Felis domesticus and Canis familiaris) obtained from IPI/ASAC Brasil, Brazil. A mean wheal $3 \mathrm{~mm}$ in diameter larger than the negative control (diluent) was considered to be positive. In parallel, blood samples $(5 \mathrm{~mL})$ were collected from all individuals and the serum was stored at $-20^{\circ} \mathrm{C}$.

\section{Mite extracts}

Total extracts of Bt, Dp, and Df were obtained from mite bodies and feces as described elsewhere (18). Briefly, mite powder was triturated in liquid nitrogen and allergens were extracted in $5 \mathrm{mM}$ borate-buffered saline containing protease inhibitors. After centrifugation $(20,000 \mathrm{~g}$ for 45 $\min$ at $4^{\circ} \mathrm{C}$ ), the supernatant was dialyzed and protein concentration was determined by the method of Lowry et al. (19).

\section{ConA-binding components from total extracts of B. tropicalis}

ConA-binding components from the Bt-total extract were obtained by affinity chromatography using ConASepharose (Amersham Pharmacia Biotech, Sweden) as described (9). After elution with $50 \mathrm{mM} \alpha$-D-methylmannopyranoside (Ferro Pfanstiehl Laboratories Inc., USA), ConAbound (Bt-ConA) fractions were concentrated, dialyzed and the protein content was determined.

\section{Measurement of specific IgE to $B$. tropicalis}

Levels of specific IgE to $B$. tropicalis and its ConAbinding fractions were measured by ELISA as described (9). Briefly, plates were coated with Bt-total or Bt-ConA extracts (1 $\mu \mathrm{g} /$ well), blocked with PBS containing 0.05\% Tween 20 and 1\% bovine serum albumin (PBST-BSA), and incubated with serum samples diluted 1:2 in PBSTBSA. After washing, plates were incubated with biotinylated anti-human IgE (Kierkegaard and Perry Lab., USA) and subsequently with streptavidin-peroxidase (Sigma,

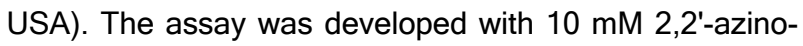
bis-(3-ethyl-benzthiazoline) sulfonic acid (ABTS; Sigma, USA) and $0.03 \% \mathrm{H}_{2} \mathrm{O}_{2}$. Absorbance was determined in a plate reader at $405 \mathrm{~nm}$ up to a maximum absorbance value of 1.5 for which the assay was linear in positive control 
sera. Antibody titers were reported as ELISA index (EI) and determined as follows: $\mathrm{EI}=$ absorbance test sample/ cutoff, where cutoff was calculated as the mean absorbance of 3 negative control sera plus 5 standard deviations, as described elsewhere (9). El values $>1.2$ were considered to be positive in order to exclude borderline reactivity values close to $\mathrm{El}=1.0$.

\section{Measurement of total serum IgE}

Total serum IgE was measured with a monoclonal antibody-based ELISA (20). Briefly, plates were coated with monoclonal anti-human IgE (1:5000; Sigma), blocked with PBST-BSA and subsequently incubated with serum $(1: 5,1: 50$ and 1:500) and biotinylated goat anti-human IgE $(1: 4000)$. Subsequent steps were similar to specific IgEELISA. Results are reported as international units per milliliter $(\mathrm{IU} / \mathrm{mL})$ and calculated on the basis of a standard curve constructed with a reference serum that contained $3000 \mathrm{IU} / \mathrm{mL}$ of total $\mathrm{lgE}$.

\section{Cellular immune response}

Twenty-six atopic patients with positive SPT to $\mathrm{Bt}, \mathrm{Dp}$, and $\mathrm{Df}$ (Bt+ group), 19 atopic patients with negative SPT to Bt, but positive SPT to Dp and Df (Bt- group), and 24 nonatopic subjects with negative SPT to all tested aeroallergens (NA group) were selected to evaluate the cellular immune response to $B$. tropicalis.

\section{Proliferation assays}

PBMC were freshly isolated from $30 \mathrm{~mL}$ heparinized blood samples by density gradient centrifugation over Ficoll-Hypaque solution (Amersham Pharmacia). PBMC were cultured in triplicate $\left(2 \times 10^{5}\right.$ cells $/ 200 \mu \mathrm{L}$ per well $)$ in 96-well plates in RPMI 1640 medium supplemented with $10 \%$ human $A B$ serum (Sigma) and stimulated with Bt-total $(10 \mu \mathrm{g} / \mathrm{mL})$ or Bt-ConA $(5 \mu \mathrm{g} / \mathrm{mL})$ extracts. Control cultures were incubated with medium alone, or mitogens (phytohemagglutinin (PHA) at $10 \mu \mathrm{g} / \mathrm{mL}$ or ConA at $5 \mu \mathrm{g} / \mathrm{mL}$ ), or non-allergen control (tetanus toxoid (TT) at $10 \mu \mathrm{g} / \mathrm{mL}$ ). Cells were incubated at $37^{\circ} \mathrm{C}$ and $5 \% \mathrm{CO}_{2}$ for 3 or 5 days when stimulated with mitogens or antigens, respectively. Cells were pulsed with $0.5 \mu \mathrm{Ci} /$ well of tritiated thymidine (New England Nuclear, USA) for the final $8 \mathrm{~h}$ of culture, harvested on glass fiber filters, and counted in a liquid scintillation ß-counter (Packard Tri-Carb 2100TR, USA) and results are reported as counts per minute (cpm).

Proliferative response in the presence of ConA inhibitor

To evaluate the interference of residual ConA in the BtConA extract on the proliferative response, PBMC were obtained from 14 patients ( 6 from $\mathrm{Bt}+$ group, 4 from Bt- group and 4 from NA group) and cultured with stimuli as described above. In parallel, the Bt-ConA extract was added in the presence of a ConA inhibitor, $\alpha$-D-methylmannopyranoside at $50 \mathrm{mM}$ as previously established in preliminary experiments. As control, ConA mitogen was also added in the presence of the ConA inhibitor. All subsequent steps were performed as described above.

\section{Cytokine production assays}

PBMC ( $1 \times 10^{5}$ cells $/ 250 \mu \mathrm{L}$ per well) were cultured in 48-well plates and stimulated with allergen extracts (Bttotal and Bt-ConA), mitogens (PHA, ConA), non-allergen control (TT) or medium alone, at the same concentrations used for the proliferation assays. Supernatant solutions were collected from independent replicate wells after 3 and 7 days of culture, and stored in aliquots at $-70^{\circ} \mathrm{C}$ until determination of cytokine levels. IFN- $\gamma$ and IL- 5 levels were measured in supernatants from cell cultures with an ELISA sandwich assay according to manufacturer instructions (R\&D Systems, USA). The sensitivity of the assays was $7.8 \mathrm{pg} / \mathrm{mL}$ for IFN- $\gamma$ and $11.7 \mathrm{pg} / \mathrm{mL}$ for IL-5. The intraand inter-assay coefficients of variations for these cytokines were below 10 and 15\%, respectively.

\section{Statistical analysis}

Statistical analysis was performed using the GraphPad Prism 4.0 software (GraphPad Software, Inc., USA). Levels of total serum $\lg E$ and specific $\lg E$ to $B$. tropicalis were compared using the Kruskal-Wallis and the Dunn multiple comparison tests. Differences between proportions were analyzed by the chi-square test. Proliferation responses and cytokine levels were compared by the Kruskal-Wallis analysis. Differences were considered to be statistically significant when $\mathrm{P}<0.05$.

\section{Results}

\section{Subject characteristics}

The demographic and clinical characteristics of the study subjects are reported in Table 1. Subjects were distributed into three groups according to SPT positivity to Bt-total extract: 1) Bt+ group: patients with positive SPT to Bt-total; 2) Bt- group: patients with negative SPT to Bt-total, but positive SPT to Dp and/or Df extract; 3) NA group: nonatopic subjects with negative SPT to all aeroallergens tested.

Rhinitis was the most frequent clinical diagnosis observed in both $\mathrm{Bt}+$ and Bt- groups, with no significant difference between the groups. The mean wheal size to Dp extract was significantly larger in the $\mathrm{Bt}+$ than the $\mathrm{Bt}-$ group, as well as in relation to Bt and Df extracts $(P<0.05)$, 
although no significant difference was found in positive SPT results (90-100\%). All NA subjects showed a negative SPT to mite extracts in addition to other aeroallergens tested (data not shown) according to the selection criteria used.

\section{SPT to $B$. tropicalis, $D$. pteronyssinus and $D$. farinae}

SPT results of 137 patients with allergic rhinitis with/ without asthma showed that $96(70.1 \%)$ patients had positive SPT to $B$. tropicalis, $95(69.4 \%)$ were simultaneously positive to 3 mites ( $\mathrm{Bt}+/ \mathrm{Dp}+/ \mathrm{Df}+)$, and only 1 patient $(0.7 \%)$ was positive to both $\mathrm{Bt}$ and $\mathrm{Df}$ extracts $(\mathrm{Bt}+/ \mathrm{Dp}-/ \mathrm{Df}+)$. No patient was monosensitized to $B$. tropicalis (Bt+/Dp-/Df-). On the other hand, of $41(29.9 \%)$ patients with negative SPT to $B$. tropicalis, 37 (27\%) were reactive to both Dp and Df extracts (Bt-/Dp+/Df+) and only 4 (2.9\%) showed reactivity to Dp extract alone (Bt-/Dp+/Df-).

Levels of specific IgE to $B$. tropicalis and total serum IgE

Levels of specific IgE to both Bt-total and Bt-ConA extracts (Figure $1 \mathrm{~A}$ ) were significantly higher in the $\mathrm{Bt}+$ group ( $E I=1.73$ and 1.20 , respectively) than in the Btgroup $(\mathrm{EI}=0.86$ and 0.84 , respectively) or the NA group (EI

Table 1. Demographic and clinical characteristics of the study subjects.

\begin{tabular}{lccc}
\hline Characteristics & \multicolumn{3}{c}{ Groups } \\
\cline { 2 - 4 } & $\mathrm{Bt}+$ & $\mathrm{Bt}-$ & $\mathrm{NA}$ \\
\hline Number of subjects (N) & 96 & 41 & 109 \\
Age (years, mean \pm SD) & $27.3 \pm 9.5$ & $28.3 \pm 9.2$ & $31.1 \pm 11.7$ \\
Gender (male/female) & $40 / 56$ & $14 / 27$ & $37 / 72$ \\
Clinical diagnosis (N, \%) & & & \\
Rhinitis & $78(81.3 \%)^{\mathrm{a}}$ & $37(90.2 \%)^{\mathrm{a}}$ & 0 \\
Rhinitis + asthma & $18(18.7 \%)^{\mathrm{b}}$ & $4(9.8 \%)^{\mathrm{b}}$ & 0 \\
& & & \\
Positive SPT (mean wheal size, $\mathrm{mm}$ ) and & positivity (\%) & 0 \\
B. tropicalis (Bt) & $7.4 \pm 3.0^{\mathrm{a}}$ & 0 & 0 \\
& $100 \% \%^{\mathrm{a}}$ & 0 & 0 \\
D. pteronyssinus (Dp) & $9.8 \pm 3.4^{\mathrm{b}}$ & $7.5 \pm 2.7^{\mathrm{a}}$ & 0 \\
& $99 \%^{\mathrm{a}}$ & $100 \%{ }^{\mathrm{a}}$ & 0 \\
D. farinae (Df) & $7.9 \pm 2.8^{\mathrm{a}}$ & $7.3 \pm 2.6^{\mathrm{a}}$ & 0 \\
& $100 \% \%^{\mathrm{a}}$ & $90 \%{ }^{\mathrm{a}}$ & 0 \\
\hline
\end{tabular}

$\mathrm{Bt}+=$ patients with positive skin prick test (SPT) to $B$. tropicalis extract; $\mathrm{Bt}-=$ patients with negative SPT to $B$. tropicalis extract; NA = non-atopic subjects. Comparisons are done horizontally between subject groups and vertically between the parameters analyzed (clinical diagnosis and positive SPT) within each subject group. a,bDifferent letters indicate statistically significant differences between mean wheal size as determined by the Kruskal-Wallis and Dunn multiple comparison tests, and between percentages of clinical diagnosis or positive SPT as determined by the chi-square test with Yates' correction or the Fisher exact probability test, when appropriate $(P<0.05)$.
$=0.78$ and 0.78 , respectively; $\mathrm{P}<0.001)$. From all $\mathrm{Bt}+$ patients, $73 \%$ had specific serum IgE to Bt-total extract and $50 \%$ to Bt-ConA extract by ELISA, with a significant difference between the seropositivity rates $(P=0.0013)$. In contrast, Bt- patients and NA subjects had no serum IgE reactivity to either Bt-total or Bt-ConA extracts. Sera assayed in plates previously coated with Con-A (negative control for the Bt-ConA extract) did not show any reactivity, with El values below 1.2 (data not shown).

Levels of total serum IgE measured by ELISA in $\mathrm{Bt}+$ $(196.2 \mathrm{IU} / \mathrm{mL})$ and Bt- $(105.8 \mathrm{IU} / \mathrm{mL})$ atopic patients were significantly higher than those detected in non-atopic subjects $(21.7 \mathrm{IU} / \mathrm{mL} ; \mathrm{P}<0.001)$, but with no significant difference between the atopic patients $(P>0.05)$.

\section{Proliferative responses}

Bt-total allergen-induced cellular proliferative responses were significantly higher compared to negative control (medium alone) only for $\mathrm{Bt}+$ patients, while Bt-ConA extract induced increased proliferative responses in all groups $(\mathrm{P}<0.05$; Figure 2). TT antigen-induced proliferative responses were significantly elevated for both groups of atopic patients $(\mathrm{P}<0.05)$. Positive controls (PHA and ConA) showed 30 - to 50-fold higher proliferation rates in relation to medium alone (Figure 2, inset). Comparing the groups, Bt-total extract induced a significantly higher proliferative response in $\mathrm{Bt}+$ patients than in Bt- and NA subjects $(P<0.001)$. In contrast, BtConA showed significantly increased cellular response in the $\mathrm{Bt}+$ group in relation to the NA group $(\mathrm{P}<0.01)$. Both $\mathrm{Bt}+$ and $\mathrm{Bt}-$ atopic patients showed significantly elevated proliferative responses after TT stimulation compared to NA subjects $(P<0.05)$.

\section{Proliferative response in the presence of ConA inhibitor}

The Bt-total extract again induced a significant proliferative response in $\mathrm{Bt}+$ patients compared to medium alone, while the Bt-ConA extract once more induced increased proliferative responses in all groups $(P<0.05$; Figure 3$)$. However, in the presence of ConA inhibitor, the Bt-ConA extract-induced responses were significantly decreased $(P<0.05)$. When comparing the groups, only the Bt-total extract induced significantly increased proliferative responses in $\mathrm{Bt}+$ patients compared to Bt- and NA subjects $(P<0.05)$. TT antigen-induced proliferative responses were also reproducible and significantly increased in both atopic patients $(P<0.05)$. Positive controls (PHA and ConA) showed significantly higher proliferation rates compared to medium while a sig- 
nificant decreased proliferative response was seen only for the ConA mitogen in the presence of its specific inhibitor (Figure 3, inset).

\section{Cytokine assays}

No significant difference was observed in IFN- $\gamma$ pro- duction after any stimulus among the patient groups, except for the Bt-ConA extract that significantly induced increased IFN- $\gamma$ levels in $\mathrm{Bt}+$ patients compared to medium alone $(P<0.05$; Figure 4$)$. Positive controls increased IFN- $\gamma$ production significantly in relation to medium in all subject groups $(P<0.05$; Figure 4 , inset).
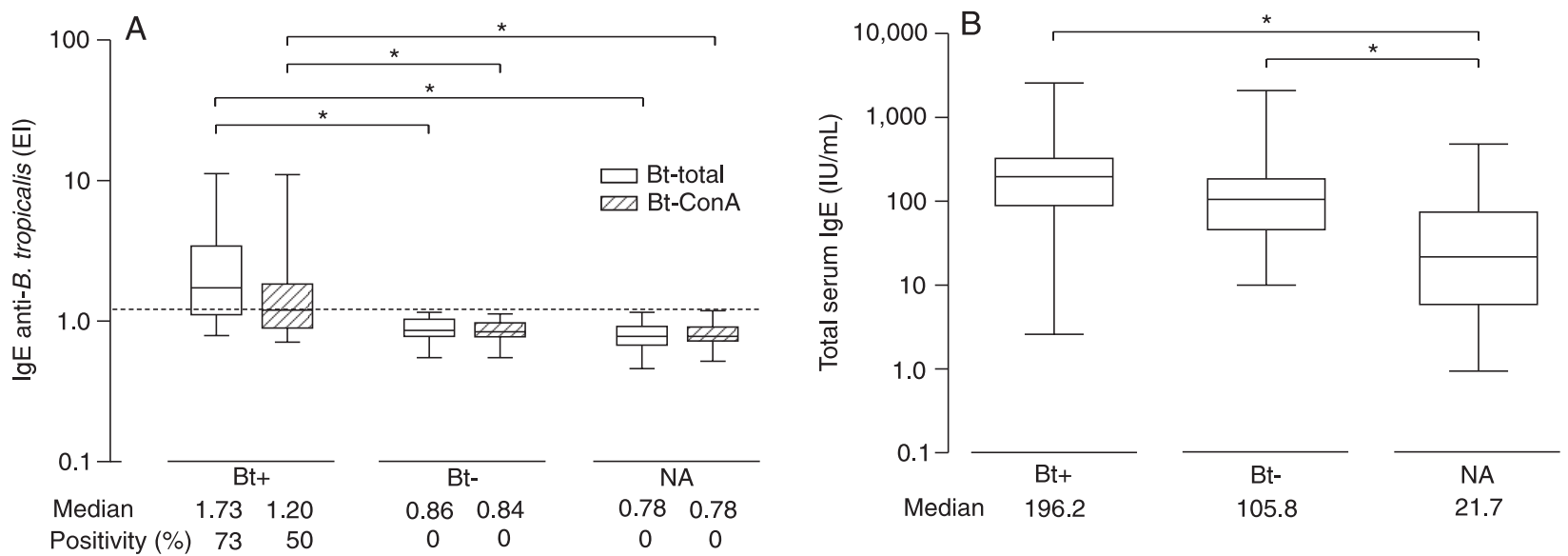

Figure 1. Levels of specific $\lg E(A)$ to Blomia tropicalis total extract (Bt-total) and concanavalin A-binding fraction (Bt-ConA), and total $\lg \mathrm{E}(\mathrm{B})$ in serum samples from patients with positive $(\mathrm{Bt}+, \mathrm{N}=96)$ or negative $(\mathrm{Bt}-, \mathrm{N}=41)$ skin prick test to $B$. tropicalis and from nonatopic subjects (NA, $N=109$ ). The dashed line indicates the cutoff of the assay (ELISA index, EI $=1.2$ ). ${ }^{*} P<0.001$ for comparisons indicated by the horizontal lines (Kruskal-Wallis and Dunn multiple comparison tests). The scales of the ordinates are logarithmic.

Figure 2. Proliferative response of peripheral blood mononuclear cells from 26 atopic patients with positive skin prick test (SPT) to Blomia tropicalis $(\mathrm{Bt}+), 19$ atopic patients with negative SPT to $B$. tropicalis (Bt-) and 24 non-atopic (NA) subjects. Cells were stimulated with $B$. tropicalis total extract (Bt-total), or concanavalin Abinding fraction (Bt-ConA), or tetanus toxoid (TT) as non-allergen control. Positive and negative controls included mitogens (phytohemagglutinin, PHA or ConA) and medium alone, respectively (inset). Data are reported as means $\pm S D$. * $P<0.05$ for comparisons between the groups indicated by the horizontal line. ${ }^{+} \mathrm{P}<0.05$ compared to medium alone (KruskalWallis and Dunn multiple comparison tests). The scales of the ordinates are logarithmic.

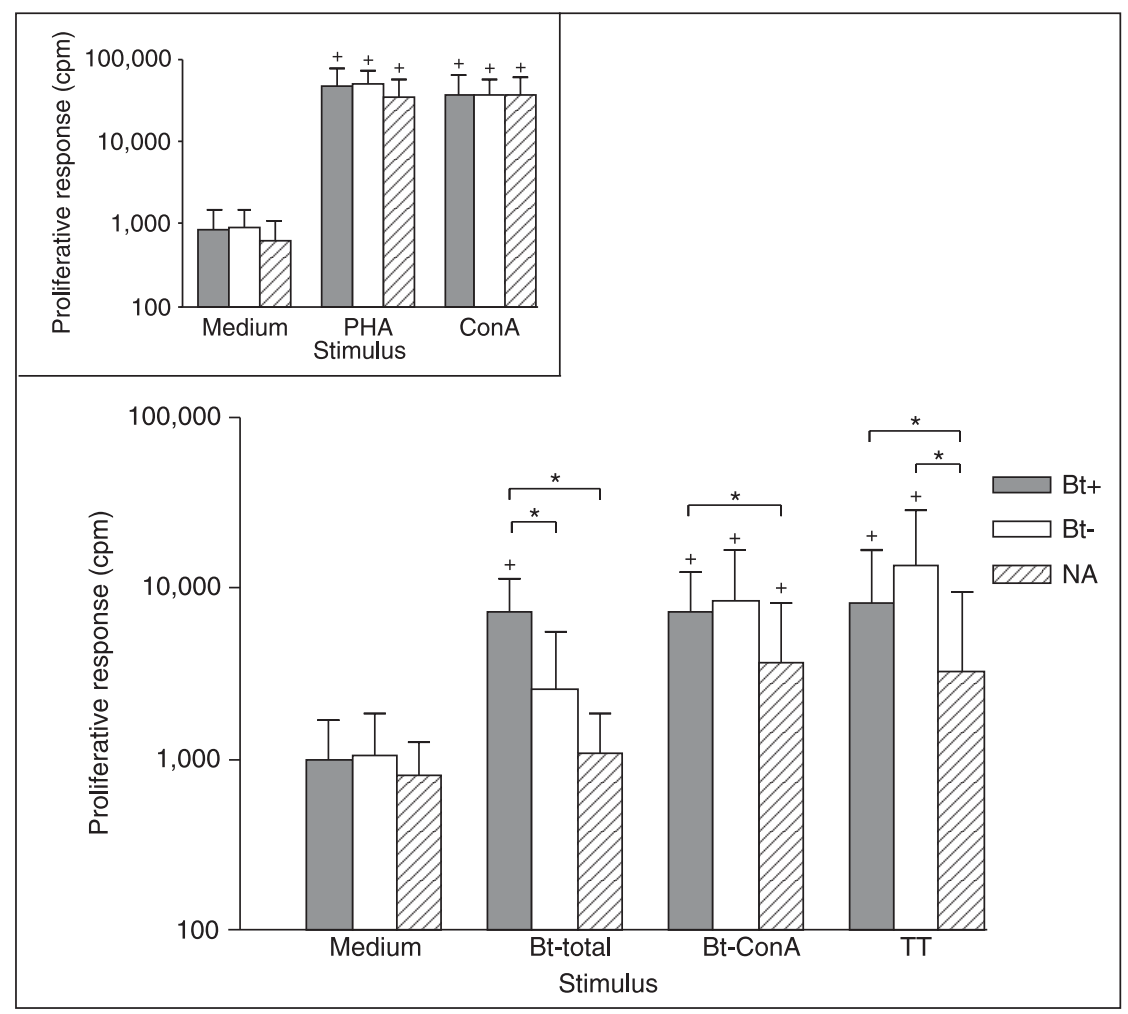


Figure 3. Proliferative response of peripheral blood mononuclear cells from 6 atopic patients with positive skin prick test (SPT) to Blomia tropicalis $(\mathrm{Bt}+), 4$ atopic patients with negative SPT to $B$. tropicalis (Bt-) and 4 non-atopic (NA) subjects. Cells were stimulated with $B$. tropicalis total extract (Bttotal), or concanavalin A-binding fraction ence of methyl- $\alpha$-D-mannopyranoside (BtConA + methyl), or tetanus toxoid (TT) as non-allergen control. Positive and negative controls included mitogens (phytohemagglutinin, PHA or ConA) and medium alone, respectively (inset). Data are reported as means \pm SD. Different uppercase letters stimuli for each group; different lowercase letters indicate significant differences between the groups for each stimulus $(P<$ 0.05 , Kruskal-Wallis and Dunn multiple comparison tests). The scales of the ordinates are logarithmic. (Bt-ConA), or Bt-ConA extract in the presindicate significant differences between the

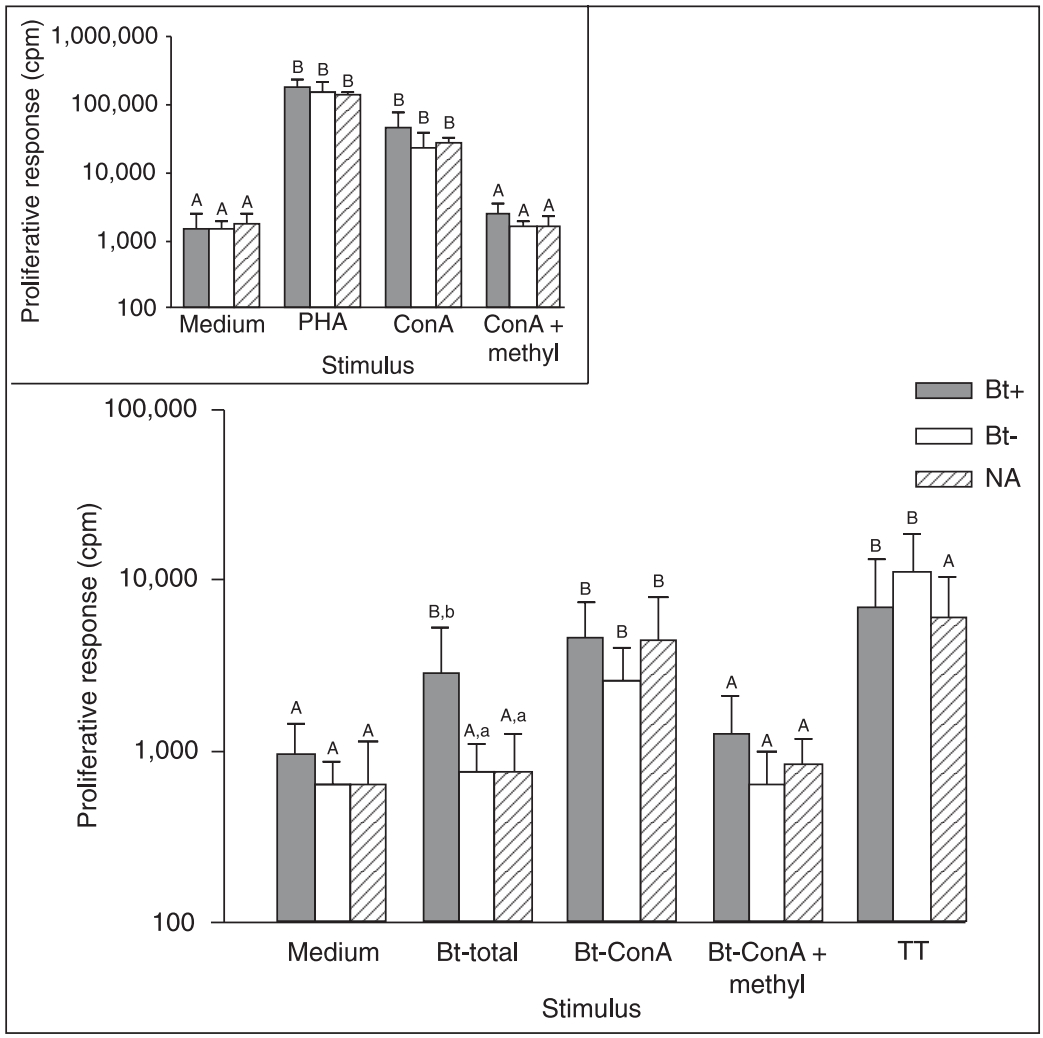

Figure 4. IFN- $\gamma$ levels in cell culture supernatants from 26 atopic patients with positive skin prick test (SPT) to Blomia tropicalis $(\mathrm{Bt}+), 19$ atopic patients with negative SPT to $B$. tropicalis (Bt-) and 24 non-atopic (NA) subjects. Cells were stimulated with $B$. tropicalis total extract (Bt-total), or concanavalin A-binding fraction (Bt-ConA), or tetanus toxoid (TT) as non-allergen control. Positive and negative controls included mitogens (phytohemagglutinin, PHA or ConA) and medium alone, respectively (inset). Data are reported as means $\pm S D$. *Significant differences in relation to medium alone $(P<0.05$, Kruskal-Wallis and Dunn multiple comparison tests). The scales of the ordinates are logarithmic.
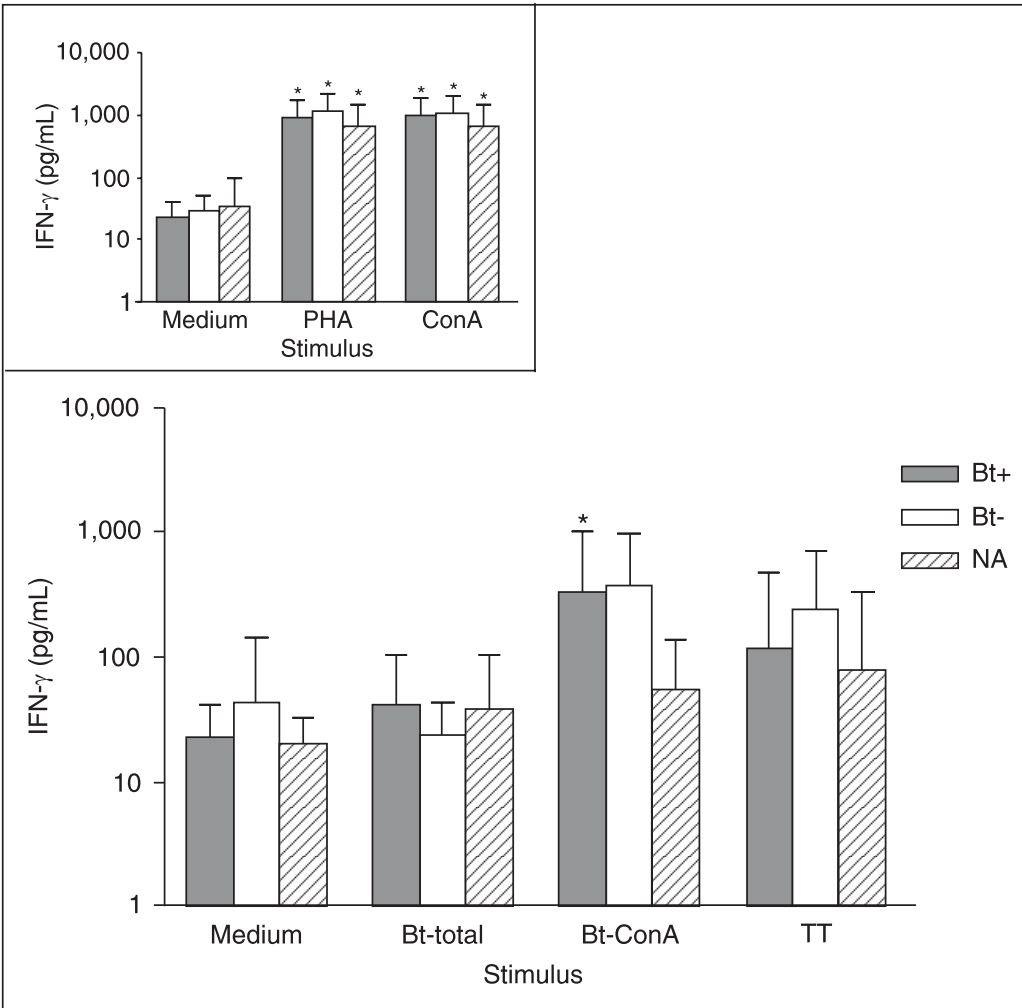
Figure 5. IL-5 levels in cell culture supernatants from 26 atopic patients with positive skin prick test (SPT) to Blomia tropicalis $(\mathrm{Bt}+), 19$ atopic patients with negative SPT to $B$. tropicalis (Bt-) and 24 non-atopic (NA) subjects. Cells were stimulated with $B$. tropicalis total extract (Bt-total), or concanavalin A-binding fraction (Bt-ConA), or tetanus toxoid (TT) as non-allergen control. Positive and negative controls included mitogens (phytohemagglutinin, PHA or ConA) and medium alone, respectively (inset). Data are reported as means $\pm S D$. ${ }^{*} P<0.05$ for comparisons between the groups for each stimulus. ${ }^{+}$Significant differences in relation to medium alone $(P<0.05$, Kruskal-Wallis and Dunn multiple comparison tests). The scales of the ordinates are logarithmic.

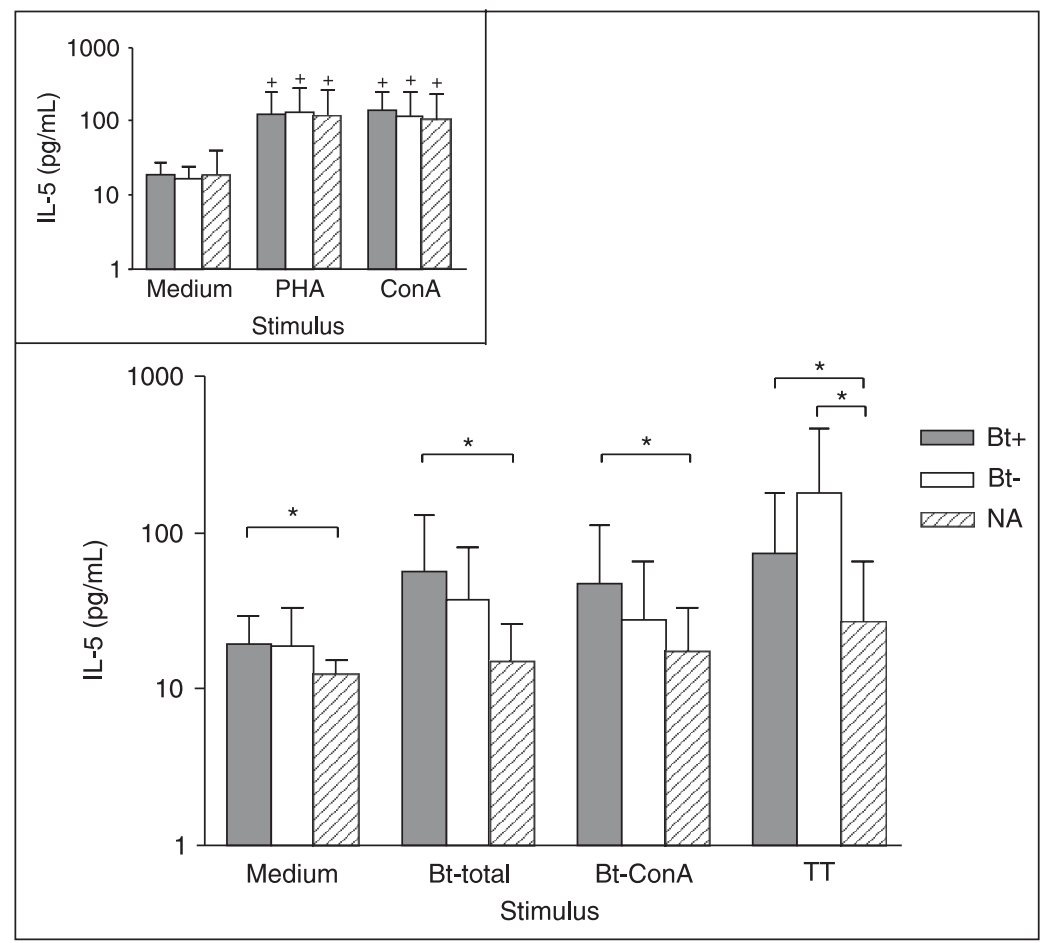

IL-5 levels were not significantly different after any stimulus in relation to medium alone, but significantly higher IL- 5 levels were detected in the $\mathrm{Bt}+$ patients compared to the NA subjects for any stimulus analyzed $(P<0.05$; Figure 5). IL-5 levels were also significantly higher in Bt- patients after stimulation with the non-allergen control (TT) in relation to NA group ( $P<0.01$; Figure 5$)$. As expected, positive controls increased IL-5 production significantly in relation to medium in all subject groups ( $P<0.05$; Figure 5 , inset).

\section{Discussion}

In the present study, we investigated the cellular and humoral immune responses to $B$. tropicalis allergens and their ConA-binding fractions in mite-sensitized patients. As expected, the great majority $(70 \%)$ of patients with allergic rhinitis with/without asthma was sensitized to $B$. tropicalis, with nearly all patients (69\%) presenting positive SPT concomitantly to the three mites (B. tropicalis, $D$. pteronyssinus, and $D$. farinae) and no patient was monosensitized to $B$. tropicalis. These results were similar to those of our previous studies carried out with another group of atopic patients from the same region $(9,18)$, confirming the low sensitization to $B$. tropicalis only and that a concurrent sensitization seems to occur with the three mites, even though a need for previous sensitization to other mite allergens cannot be ruled out. Therefore, this concomitant sensitization has complicated the evaluation of the role of $B$. tropicalis in these patients, particularly in this geographic region in which these mites have commonly been found. In addition, the sensitization to Dermatophagoides spp seems to be a major mechanism in the pathogenesis of respiratory allergic diseases in Brazil.

Similar levels of specific IgE to Bt-total and Bt-ConA extracts were found among $\mathrm{Bt}+$ patients, supporting our previous reports (9) that Bt-ConA extract contains clinically relevant allergens responsible for the induction of $\mathrm{IgE}$ antibodies. In addition, patients non-sensitized to $B$. tropicalis or non-atopic subjects showed no reactivity to both extracts, confirming the high specificity of the assay. Despite the similar levels of specific $\operatorname{lgE}$, seropositivity rates to Bt-total and Bt-ConA extracts were significantly different, indicating that the glycosylated components are not uniformly recognized by atopic patients and that the fractionation of the total extract could have contributed to the lack of some relevant sensitizing epitopes. As expected, the total serum IgE levels were found in both groups of atopic patients, regardless of the mite sensitizing agents, B. tropicalis and/or Dermatophagoides spp. Thus, despite the detection of total serum $\mathrm{IgE}$, which is not able to discriminate among different atopic patients, IgE has been 
shown to be a useful laboratory parameter for differentiating atopic from non-atopic subjects. Taken together, ELISA for the detection of $B$. tropicalis-specific $\lg E$ antibodies and IgG subclasses might be used to obtain consistent and reproducible results and it can be considered as an additional method for evaluating the humoral immune response to $B$. tropicalis in allergic patients.

Significant PBMC proliferation, a measure of the cellular immune response, was observed in response to Bttotal allergen extract in $B$. tropicalis-sensitized $(\mathrm{Bt}+)$, but not in non-sensitized (Bt-) patients and non-atopic individuals. This indicates that the allergic response involves not only specific IgE production to allergens with histamine release, but also cellular proliferation, particularly of allergen-specific $\mathrm{T}$ lymphocytes and cytokine secretion (21). In contrast, the Bt-ConA allergen extract induced increased proliferative responses in all groups of patients, even though significantly only in $\mathrm{Bt}+$ atopic patients compared to non-atopic subjects. These findings suggest a role for mannose-enriched glycosylated components present in this extract, as potential inducers of the cell proliferation. Alternatively, these data could reflect a residual effect of ConA present in the Bt-ConA extract. To clarify this latter hypothesis, cellular proliferation assays using Bt-ConA in the presence of specific ConA inhibitor showed that these responses were significantly decreased, reinforcing the presence of ConA residues in the Bt-ConA extract. It is noteworthy that the Bt-ConA extract was probed with polyclonal mouse anti-ConA antibodies in ELISA and no specific reactivity was observed (data not shown). Therefore, further studies are required for improving the ConA-Sepharose column cross-linking in order to remove residual ConA and subsequently to evaluate the actual role of these mannose-enriched glycosylated antigens.

Little is known about the cellular immune response to B. tropicalis. Recently, Lozano et al. (22) evaluated the
PBMC proliferative response to Bt allergen extract and BtM recombinant protein, demonstrating that recombinant allergens are also able to induce cellular immune responses similar to native allergen-induced responses, with potential applications in immunotherapeutic procedures. In the present study, a significant IFN- $\gamma$ production, a Th1 typical cytokine, was observed only after Bt-ConA stimulation in B. tropicalis-sensitized patients while Bt-total allergen extract showed no significant change in this cytokine production. On the other hand, significant IL-5 production, a Th2 typical cytokine, was consistently seen in B. tropicalis allergic patients under specific allergen stimulation (Bttotal and Bt-ConA) as well as unrelated antigen (TT) and even with no stimulus (medium alone), indicating that PBMC from allergic patients are prone to produce Th2 profile cytokines, spontaneously or inductively by allergen restimulation. In our previous study (9), Bt-ConA extract components were recognized by specific IgG1 antibodies (Th1 profile) rather than IgG4 antibodies (Th2 profile) in serum samples from $B$. tropicalis-sensitized patients, probably reflecting its ability to induce a protective immune response through the development of Th1 cells. Altogether, it can be concluded that ConA-binding components isolated from $B$. tropicalis may contain relevant antigens that are involved in both humoral and cellular immune responses. However, without an additional purification procedure to eliminate the residual contamination with ConA, its use in immunotherapeutic procedures cannot be recommended.

\section{Acknowledgments}

We thank Dr. Federico Montealegre-Golcher, Ponce School of Medicine, Ponce, USA, for providing dried samples of $B$. tropicalis and $D$. pteronyssinus.

\section{References}

1. Arlian LG, Bernstein D, Bernstein IL, Friedman S, Grant A, Lieberman $P$, et al. Prevalence of dust mites in the homes of people with asthma living in eight different geographic areas of the United States. J Allergy Clin Immunol 1992; 90: 292300.

2. Fernandez-Caldas E, Puerta L, Mercado D, Lockey RF, Caraballo LR. Mite fauna, Der $\mathrm{p} \mathrm{I}$, Der $\mathrm{fI}$ and Blomia tropicalis allergen levels in a tropical environment. Clin Exp Allergy 1993; 23: 292-297.

3. Arruda LK, Rizzo MC, Chapman MD, Fernandez-Caldas E, Baggio D, Platts-Mills TA, et al. Exposure and sensitization to dust mite allergens among asthmatic children in São
Paulo, Brazil. Clin Exp Allergy 1991; 21: 433-439.

4. Arruda LK, Vailes LD, Platts-Mills TA, Fernandez-Caldas E, Montealegre F, Lin KL, et al. Sensitization to Blomia tropicalis in patients with asthma and identification of allergen Blo $\mathrm{t}$ 5. Am J Respir Crit Care Med 1997; 155: 343-350.

5. Puccia R, Travassos LR, Rodrigues EG, Carmona AK, Oliveira MC, Juliano $L$. Purification of the specific exocellular antigen gp43 from Paracoccidioides brasiliensis: immunological and proteolytic activities. In: Mareska B, Kobayashi GS (Editors), Molecular biology of pathogenic fungi: a laboratory manual. New York: Telos Press; 1994. p 507-515.

6. Pitzurra L, Vecchiarelli A, Peducci R, Cardinali A, Bistoni F. 
Identification of a 105 kilodalton Cryptococcus neoformans mannoprotein involved in human cell-mediated immune response. J Med Vet Mycol 1997; 35: 299-303.

7. Gomez BL, Figueroa JI, Hamilton AJ, Diez S, Rojas M, Tobon A, et al. Detection of the 70-kilodalton histoplasma capsulatum antigen in serum of histoplasmosis patients: correlation between antigenemia and therapy during followup. J Clin Microbiol 1999; 37: 675-680.

8. Savolainen J. A standardized densitometric immunoblotting analysis of Candida albicans protein allergens. Clin Exp Allergy 1995; 25: 357-363.

9. Almeida KC, Silva DA, Gennari-Cardoso ML, Cunha-Junior JP, Alves R, Ynoue LH, et al. Responses of IgE, IgG1, and IgG4 to concanavalin A-binding Blomia tropicalis antigens in allergic patients. Braz J Med Biol Res 2006; 39: 14451454.

10. Tsai JJ, Wu HH, Shen HD, Hsu EL, Wang SR. Sensitization to Blomia tropicalis among asthmatic patients in Taiwan. Int Arch Allergy Immunol 1998; 115: 144-149.

11. Caraballo L, Puerta L, Martinez B, Moreno L. Identification of allergens from the mite Blomia tropicalis. Clin Exp Allergy 1994; 24: 1056-1060

12. Ngoc PL, Gold DR, Tzianabos AO, Weiss ST, Celedon JC. Cytokines, allergy, and asthma. Curr Opin Allergy Clin Immunol 2005; 5: 161-166.

13. Romagnani $\mathrm{S}$. Lymphokine production by human $\mathrm{T}$ cells in disease states. Annu Rev Immunol 1994; 12: 227-257.

14. Akdis CA, Blaser K, Akdis M. Genes of tolerance. Allergy 2004; 59: 897-913.

15. Bousquet J, Khaltaev N, Cruz AA, Denburg J, Fokkens WJ,
Togias A, et al. Allergic Rhinitis and its Impact on Asthma (ARIA) 2008 update (in collaboration with the World Health Organization, GA(2)LEN and AllerGen). Allergy 2008; 63 (Suppl 86): 8-160.

16. IV Diretrizes Brasileiras para o Manejo da Asma. Rev Bras Alergia Imunopatol 2006; 29: 222-245.

17. Sociedade Brasileira de Pneumologia e Tisiologia. III Consenso Brasileiro de Manejo da Asma. J Pneumol 2002; 28: 1-28.

18. Pereira EA, Silva DA, Cunha-Junior JP, Almeida KC, Alves $\mathrm{R}$, Sung SJ, et al. IgE, IgG1, and IgG4 antibody responses to Blomia tropicalis in atopic patients. Allergy 2005; 60: 401406.

19. Lowry $\mathrm{OH}$, Rosebrough NJ, Farr AL, Randall RJ. Protein measurement with the Folin phenol reagent. $\mathrm{J} \mathrm{Biol} \mathrm{Chem}$ 1951; 193: 265-275.

20. Silva DA, Gervasio AM, Sopelete MC, Arruda-Chaves E, Arruda LK, Chapman MD, et al. A sensitive reverse ELISA for the measurement of specific IgE to Der p 2, a major Dermatophagoides pteronyssinus allergen. Ann Allergy Asthma Immunol 2001; 86: 545-550.

21. Valenta R, Sperr WR, Ferreira F, Valent P, Sillaber C, Tejkl $\mathrm{M}$, et al. Induction of specific histamine release from basophils with purified natural and recombinant birch pollen allergens. J Allergy Clin Immunol 1993; 91: 88-97.

22. Lozano CP, Cano JM, Bonfante LH. Respuesta proliferativa de células mononucleares de sangre periférica al alergeno recombinante BtM del ácaro del polvo casero Blomia tropicalis. Allergol Immunopathol 2004; 32: 247-251. 